Night-Light Remote Sensing: Data, Processing and Applications

\title{
$\mathrm{Xi} \mathrm{Li}$
}

Wuhan University of Science and Technology, China

\section{Deren Li}

Wuhan University of Science and Technology, China

\section{Huayi Wu}

Wuhan University of Science and Technology, China

Night-light remote sensing is a tool which can be used when working towards achieving the UN Sustainable Development Goals. It has attracted a lot of attention and brought many new research directions as an emerging subject.

\subsection{Introduction}

Night-light remote sensing has attracted a lot of attention and brought many new research directions as an emerging subject. Satellites acquire night-light images by detecting visible light sources such as city lights, fishing boat lights and fire spots under cloudless conditions at night. Unlike daytime remote sensing, night-time remote sensing has unique capabilities for reflecting human activities, which can be used to study in the following fields: regional development [9, 3], conflict evaluation [17], light pollution [1], and fishery [5]. At present, night-light data is mainly obtained through sensors in the visible and near-infrared bands, mainly including Defense Meteorological Satellite Program/Operational Linescan System (DMSP/OLS) night-light, Visible Infrared Imaging Radiometer Suite (VIIRS) Day/Night Band DNB (DNB), Earth Remote Observation System-B (EROS-B), Luojia 1-01, and Jilin1-03B night-light images. Besides, photographs taken by astronauts from the International Space Station (ISS) can also reflect night light on the earth's surface, 
and other research used aircrafts to take photos to study night light for cities. Figures 17.1 to 17.4 show the night-light images from different sensors.

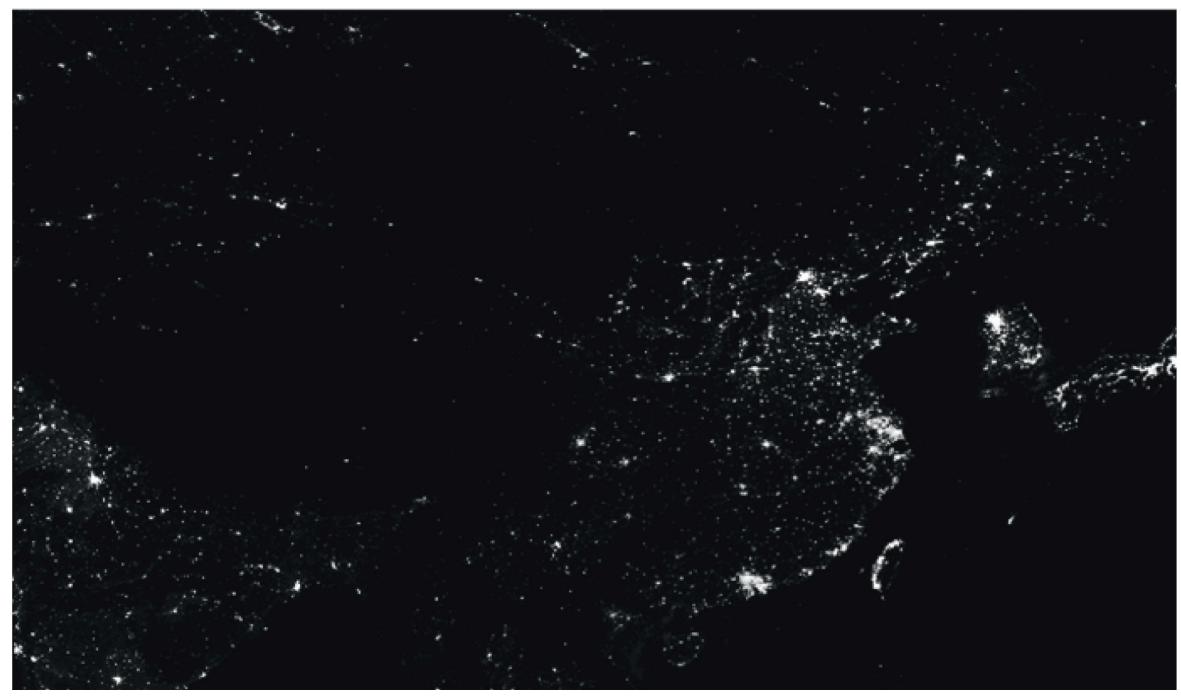

\section{FIGURE 17.1}

The DMSP/OLS night-time light image of East Asia in 2012.

\subsection{DMSP/OLS Night-light Data}

As the first night-time remote sensing satellite, DMSP has gradually been used to conduct research on night-time remote sensing. The DMSP/OLS was originally designed to detect night clouds, but scientists found that it also has the ability to detect night-time light on the earth's surface[14]. National Oceanic and Atmospheric Administration (NOAA) has released the DMSP/OLS night-light annual global composites (1992-2013) from six satellites (F10, F12, F14, F15, F16, F18) ${ }^{1}$. The spatial resolution of the image is 0.0083 degree, and the images have only digital values and no radiation units. Because the DMSP/OLS image is pressed as 6 bits digital number (DN) values, saturation commonly exists in urban cores. Therefore, DMSP/OLS images are mostly used for macroscopic research.

\footnotetext{
${ }^{1}$ https://ngdc.noaa.gov/eog/dmsp/downloadV4composites.html
} 

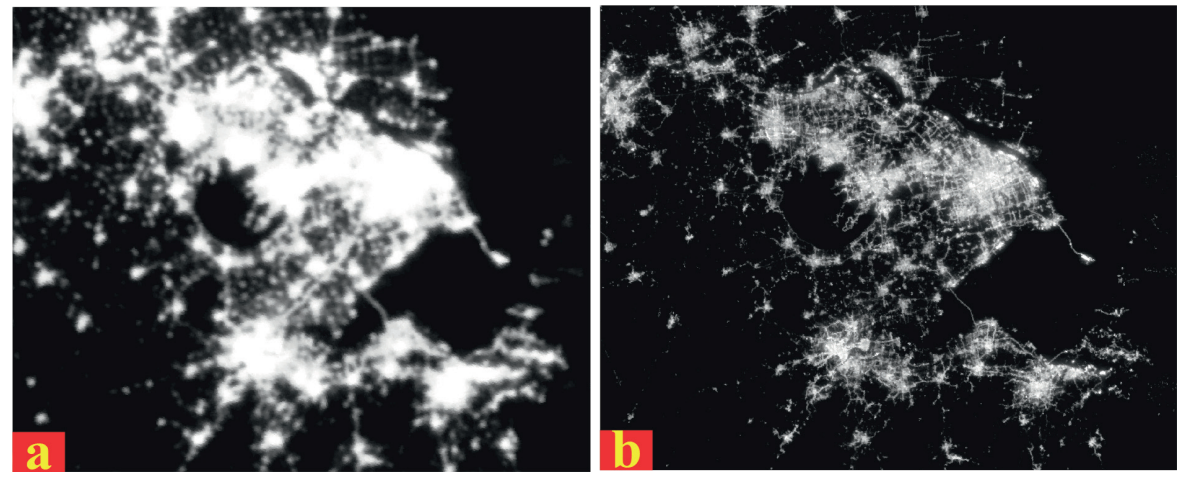

FIGURE 17.2

The (a)DMSP/OLS and (b)VIIRS DNB night-time light images of Yangtze River Delta in 2012.

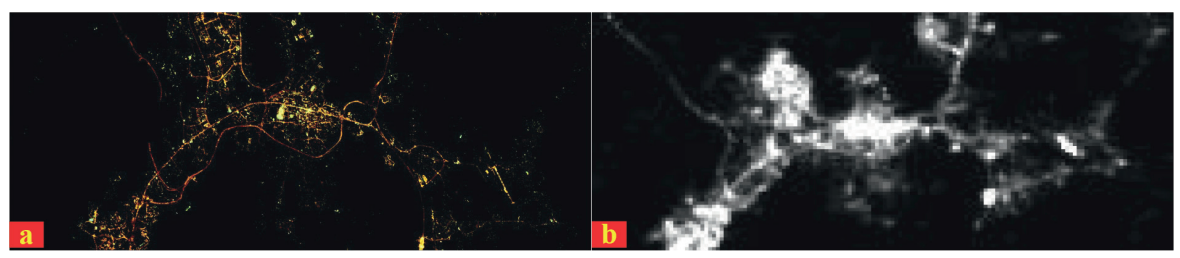

\section{FIGURE 17.3}

The (a) Jilin1-03B (June 27,2017) and (b) Luojia 1-01 (January 31, 2019) night-time light images of Hong Kong.

\subsubsection{VIIRS DNB Night-light Data}

The VIIRS was carried by Suomi National Polar-Orbiting Partnership (Suomi NPP) satellite which was launched on October 2011. VIIRS DNB has the ability to detect surface light and has higher spatial and radiation resolution than DMSP/OLS data [21]. NOAA has released three VIIRS DNB products, including daily products, monthly products and annual products (2012 - present). Its spatial resolution is $742 \mathrm{~m}$ and it can revisit most ground points in 12 hours. VIIRS DNB images can be used for quantitative research after radiation correction processing. It can be used not only for macroscopic research, but also for microscopic research on the development structure of the city [4].

\subsubsection{VIIRS DNB Night-light Data}

The EROS-B satellite was launched on April 25, 2006 from Svobodny Launch Complex in eastern Siberia. It has a panchromatic band ranging from 0.5 to $0.9 \mu \mathrm{m}$, and the scan width of the imagery was about $8.3 \mathrm{~km}$. EROS-B images have a high spatial resolution which is $0.7 \mathrm{~m}$. The data is described as 16 bits 

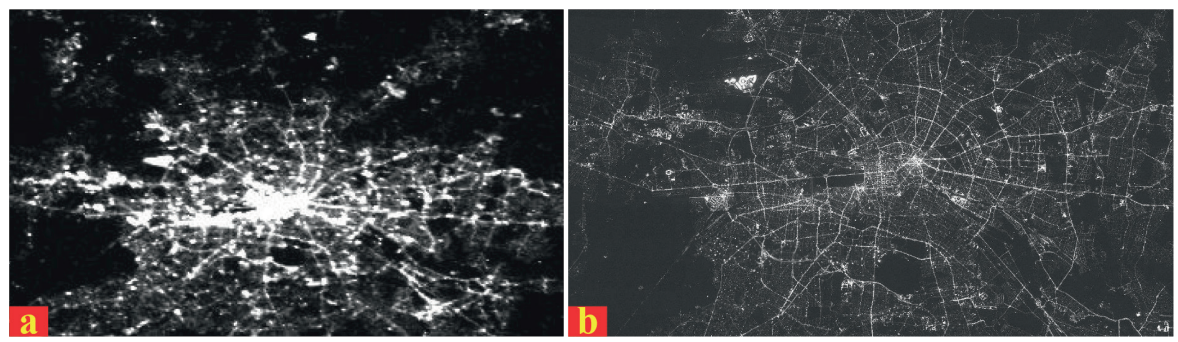

\section{FIGURE 17.4}

The (a) Luojia 1-01 (August 27, 2018) and (b) aerial survey using CCD (September 11, 2010) night-time light images of Berlin.

DN values (0 - 65535) to represent brightness values. There is no information on how to calibrate its DN values into radiance values [15].

\subsubsection{Jilin1-03B Night-light Data}

Chinese commercial satellite - Jilin1-03B was launched on January 9, 2017 by the Chinese commercial satellite company - Chang Guang Satellite Technology Co., Ltd. It has multi-spectral bands, including 430-512 nm (blue), 489-585 nm (green) and 580-720 nm (red), and has a high spatial resolution of $0.92 \mathrm{~m}$. It is the first multi-spectral bands satellite which can detect low light at $7 \mathrm{E}-7 \mathrm{~W} / \mathrm{cm}^{2} / \mathrm{sr}[28]$. Jilin1-03B data can be used to study light pollution and light distribution inside cities.

\subsubsection{Luojia 1-01 Night-light Data}

Luojia 1-01 satellite was launched on June 2, 2018. It has been equipped with a 4-megapixel scientific CMOS Image Sensor [25]. It is the first remote sensing satellite used for night light study in China and also the first low-orbit satellite with earth observation and satellite navigation enhancement functions. The image of Luojia 1-01 has a high spatial resolution $(129 \mathrm{~m})$, and a wide band range from $460 \mathrm{~nm}$ to $980 \mathrm{~nm}$. It can revisit a point in 15 days. The data can be used in socio-economic parameter estimation, eco-environmental disaster monitoring, major event assessment, public health, etc. Table 17.1 shows the comparison of different night-light remote sensing satellites. 
TABLE 17.1

The comparison of different satellites.

\begin{tabular}{|c|c|c|c|c|c|}
\hline Variable & $\begin{array}{l}\text { DMSP / } \\
\text { OLS }\end{array}$ & $\begin{array}{l}\text { VIIRS } \\
\text { DNB }\end{array}$ & EROS-B & $\begin{array}{l}\text { Jilin } \\
\text { 1-03B }\end{array}$ & $\begin{array}{l}\text { Luojia } \\
1-01\end{array}$ \\
\hline Available & $1992-2013$ & $\begin{array}{ll}2012 & - \\
\text { present }\end{array}$ & $\begin{array}{ll}2006 & - \\
\text { present }\end{array}$ & $\begin{array}{ll}2017 & - \\
\text { present }\end{array}$ & $\begin{array}{l}2018 \\
\text { present }\end{array}$ \\
\hline Country & The U.S. & The U.S. & Israel & China & China \\
\hline Swath & $3000 \mathrm{~km}$ & $3000 \mathrm{~km}$ & $8.3 \mathrm{~km}$ & $11.6 \mathrm{~km}$ & $250 \mathrm{~km}$ \\
\hline $\begin{array}{l}\text { Spatial } \\
\text { Resolution }\end{array}$ & $2.7 \mathrm{~km}$ & $742 \mathrm{~m}$ & $0.7 \mathrm{~m}$ & $0.92 \mathrm{~m}$ & $129 \mathrm{~m}$ \\
\hline Quantization & 6 bits & 14 bits & 16 bits & 8 bits & 14 bits \\
\hline Saturation & Saturated & $\begin{array}{l}\text { Not } \\
\text { Saturated }\end{array}$ & $\begin{array}{l}\text { Not } \\
\text { Saturated }\end{array}$ & $\begin{array}{l}\text { Not } \\
\text { Saturated }\end{array}$ & $\begin{array}{l}\text { Not } \\
\text { Saturated }\end{array}$ \\
\hline $\begin{array}{l}\text { Radiance } \\
\text { Calibration }\end{array}$ & No & Yes & No & Yes & Yes \\
\hline Bands $(\mu \mathbf{m})$ & $0.50-0.90$ & $0.50-0.90$ & $0.50-0.90$ & $\begin{array}{l}\text { Blue: } \\
0.43-0.51 ; \\
\text { Green: } \\
0.49-0.59 ; \\
\text { Red: } \\
0.58-0.72\end{array}$ & $0.46-0.98$ \\
\hline
\end{tabular}

\subsection{Data Processing}

\subsubsection{DMSP/OLS Intercalibration}

DMSP/OLS sensors have collected a long time series night-time light (NTL) data, which is useful in studying changes of human activities including but not limited to energy usage, population distribution and urbanization dynamic. However, due to the systematic differences in satellite orbits and sensor degradation, the NTL data collected by different sensors are very different, and as a result, its potential is not fully utilized. In order to eliminate the inconsistency of NTL data and facilitate scientific research, it is necessary to perform intercalibration to NTL data.

According to Zhang et al.[26], the existing strategies in calibrating NTL time series can be mainly grouped into two categories: 1) Manually selecting the invariant area as reference area based on experience $[12,10,23,2,20,24]$ and 2) Automatic identification of stable pixels as pseudo-invariant features [16]. Although there are many differences among all calibration methods, the two assumptions are the most critical: 1) there is very little ground change at the calibration sites, and 2) sensor deviations in different regions are consistent. For example, Elvidge et al.[10] calibrated global DMSP/OLS NTL time series using the Sicily of Italy as the calibration site, Li et al.[16] assumed that land change is an outlier and used an algorithm to iterative the outlier and 
discard them, and Zhang et al.[26] automatically identified the data points along the ridgeline and used those points to derive calibration.

We can tell from the graph below, intercalibration significantly improved the consistency of TSOL (total sum of lights).

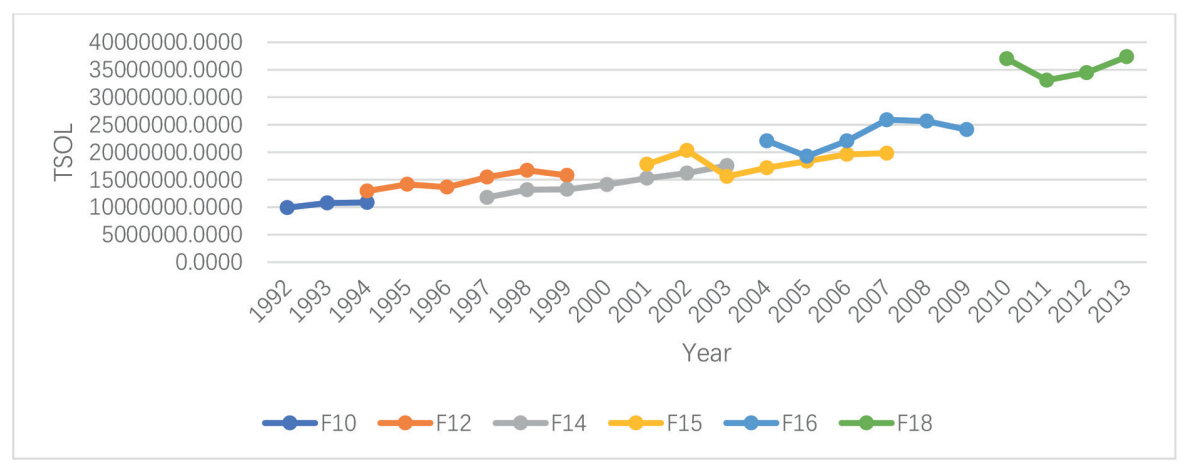

FIGURE 17.5

Sum of lights time series from raw NTL (China).

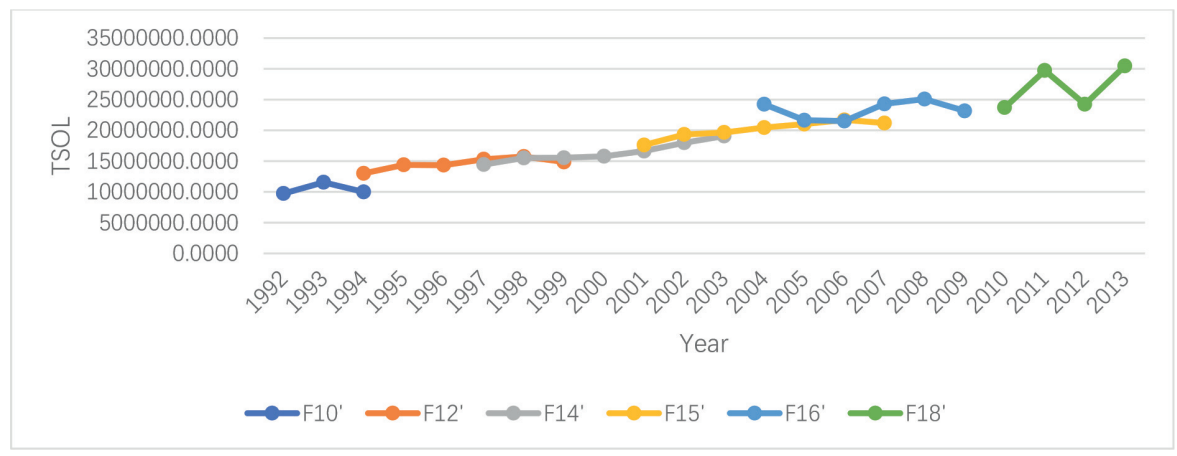

FIGURE 17.6

Sum of lights time series from intercalibrated NTL (China).

\subsubsection{Improvement of VIIRS DNB Daily Data}

The radiance of VIIRS DNB night light data is affected by moonlight, atmosphere, snow, etc. after radiation calibration, and further correction is necessary to more accurately describe the radiance of the artificial light on the surface. Moreover, since the acquisition time of some images is in the day and night, the refraction of sunlight through the atmosphere will affect the 
night light data of the night-time region. Therefore, we need to eliminate those affects and follow the method of Román and Stokes to get radiance $\mathrm{L}_{\mathrm{NTL}}$ [22].

$$
L_{N T L}=\frac{L_{D N B}-L_{p a t h}}{T_{\uparrow}}-L_{m} T_{\downarrow}
$$

where $\mathrm{L}_{\mathrm{DNB}}$ represents the radiance of the night light of the VIIRS DNB band at the top of the atmosphere, $\mathrm{L}_{\text {path }}$ represents the total radiance of the path radiation received by the satellite observation direction, $\mathrm{L}_{\mathrm{m}}$ represents the lunar radiance, $\mathrm{T}_{\uparrow}$ represents the total path transmittance of the surface to the sensor, and $\mathrm{T}_{\downarrow}$ is the total transmission rate of the moon to the surface.

\subsubsection{Saturation Correction}

When it comes to NTL data application, saturation is a very serious problem, which leads to the lack of details in urban cores in NTL imagery. As DMSP/OLS data has only 8-bit quantization and low dynamic range, saturation of data values in core urban areas limits the utility of NTL data for many applications.

In general, the correction methods of saturation fall into two categories: those that utilize only NTL data, such as linear regression method [13], and those that use other satellite data to correct the saturation of NTL data, like Vegetation Adjusted NTL Urban Index (VSNUI)[27] and Human Settlement Index (HSI) [19].

For the former category that utilize only NTL data, the main idea is based on the assumption that the tendency of DN change in the saturated area is similar to that of the non-saturated area. So we can extract the NTL of nonsaturated area, and then develop a regression model, either linearly or nonelinearly, to adjust the saturated area. And for the latter category, the main idea is that NTL has a strong relationship with human activities. A typical example is urbanization results in land cover change, specifically the loss of agricultural, natural vegetation, or abandoned lands to construct the built environment in most industrialized countries and emerging economies. Therefore, any data source about vegetation abundance and environment change can be used to generate an index to adjust the saturation of NTL data.

\subsection{Applications}

\subsubsection{The Applications of Night-light Data}

Socio-economic data often contains measurement errors and lacks spatial continuity in many developing and underdeveloped regions of the world. Nighttime light, a special tool has a positive correlation with human activities, 


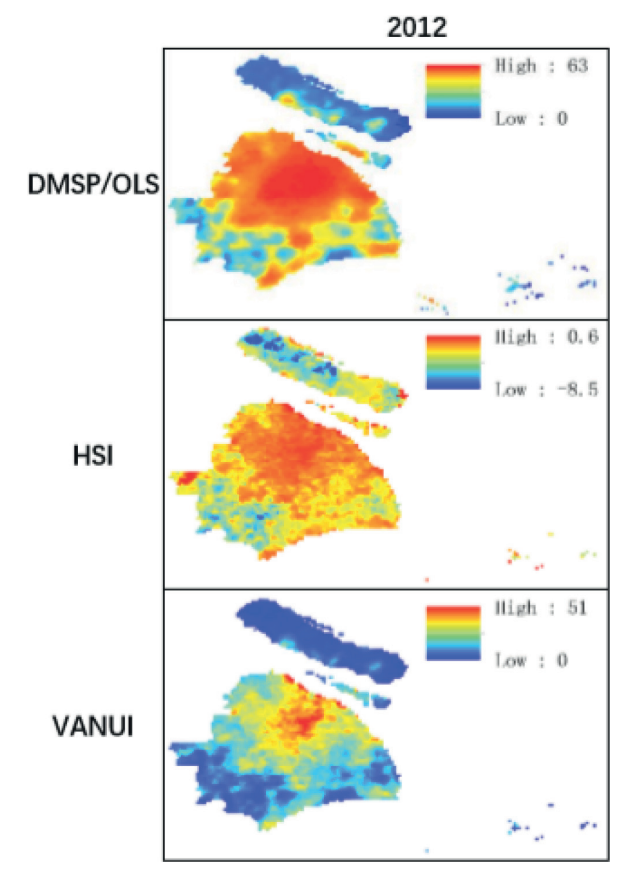

\section{FIGURE 17.7}

The spatial pattern of DMSP/OLS data, HSI and VANUI in Shanghai in 2012.

which provides potential possibilities to quantify these socio-economic data. Elvidge et al.[11] utilized the DMSP/ OLS NTL imagery of 21 countries in the America to do regression analysis, and found out that the correlation coefficient between total NTL and GDP in light-emitting area is 0.97. Since then, scientists carried on similar researches on different regions like European Union[7], China[18], etc.

All of those researches show a strong relationship between NTL and GDP. Now it is widely believed that the NTL is an efficient economic indicator in economic application, such as urbanization research, economic growth and decline analysis, and regional policy assessment. Recently, more studies reveal that NTL data is also an objective tool that can be used in the following fields.

Light pollution: Artificial night-time lighting has diverse and problematic environmental impacts. These include effects on the physiology, behavior and phenology of organisms. Sánchez de Miguel, A. et al.[6] first presented a method, color-color diagrams, to classify outdoor lighting types from ISS(International Space Station) imagery, then determined the relations between the spectral information that can be obtained from the imagery and some key environmental indices, including photopic vision, the Melatonin Suppression Index, the Star Light Index, the Induced Photosynthesis Index, pro- 
duction of $\mathrm{NO}_{2}-\mathrm{NO}$ radicals, energy efficiency and $\mathrm{CO}_{2}$ emissions, and Correlated Color Temperature.

Demography: Combination of NTL data with other auxiliary data can help to disaggregate demographic data more accurately. By combining NTL imagery, vegetation index products, and population statistics, Zhuo et al.[29] simulated the population density distribution at $1-\mathrm{km}$ resolution grids by establishing different population spatial distribution models.

Natural disaster loss assessment: Elliott, R. J. R., et al.[8] examined the impact of typhoons on local economic activity in coastal China by combining historical typhoon track data and damage proxy with satellite derived nightlight intensity data to construct a panel data set, which allows researchers to estimate the impact of typhoons at a spatially highly disaggregated level. They found that typhoons have a negative, significant, but short-term impact on local activity.

Humanitarian support: Since reliable witness reports are hard to gather in a war zone, satellite images, as one of the few sources of objective information, are potentially of great importance. Li, X. and D. Li[17] used 38 monthly DMSP/OLS composites covering the period between January 2008 and February 2014 as a NTL time series, and found that NTL and lit areas in Syria during the crisis declined by about $74 \%$ and $73 \%$ respectively. In 12 of 14 provinces, the NTL declined by $>60 \%$. These findings lend support to the hypothesis that NTL can be a useful source for monitoring humanitarian crises such as that unfolding in Syria.

\subsubsection{Case: The Study of Syrian Crisis}

At the beginning of 2011, the Syrian civil war broke out. Many Syrians were forced to flee their homes and lost their lives due to lack of food and medical resources. At present, the number of deaths in the Syrian civil war has exceeded 400,000, causing an almost devastating blow to the entire country. However, how to assess the impact of the war timely and accurately is a very important and difficult problem. Witness reports are currently the main source of war assessment, but during violent periods, the comprehensiveness and neutrality of witness reports are difficult to guarantee. Consequently, remote sensing satellite data has been used as a supplement in war assessment. The remote sensing satellite image is an objective and accurate data source, and the night-time light remote sensing image can be regarded as a representation of human activities and social economy. When the social and economic system of a certain place changes significantly due to disasters, its night light will also fluctuate greatly. Therefore, the NTL data has been widely used for assessing the impact of war.

Li et al.[17] investigated the impact of the Syrian crisis using DMSP/OLS NTL remote sensing data. Firstly, the DMSP/OLS time series images were registered, denoised and calibrated using invariant region-based intercalibration method. Then visual comparison, analysis by administrative regions and 
spatial analysis were applied to demonstrate the impact of the Syrian civil war objectively. By comparing the DMSP/OLS images in two periods, it can be seen that the night light in Syria has been sharply reduced since the outbreak of the civil war, and the lights in some towns have even disappeared completely. The change of NTL were calculated as Equation (2):

$$
S N L_{\text {change }}=\frac{S N L_{m}-S N L_{n}}{S N L_{n}}
$$

where $\mathrm{SNL}_{\text {change }}$ represents the change of NTL, $\mathrm{SNL}_{\mathrm{m}}$ and $\mathrm{SNL}_{\mathrm{n}}$ represent the sum of night lights at two different periods. According to the calculation results, the sum of night light in Syria decreased by about $74 \%$ from March 2011 to February 2014. Among the 14 provinces in Syria, 12 provinces have reduced their lights by more than $60 \%$, and the areas with the most serious night light reduction are also the most intense places in the civil war. While areas such as Damascus, controlled by Syrian government forces, have seen relatively little reduction in night light. In addition, the linear regression method was used to analyse the relationship between the reduction of night light and the number of refugees in different provinces. The study found there is a significant positive correlation, confirming that refugee migration is one of the important factors causing the reduction of night light.

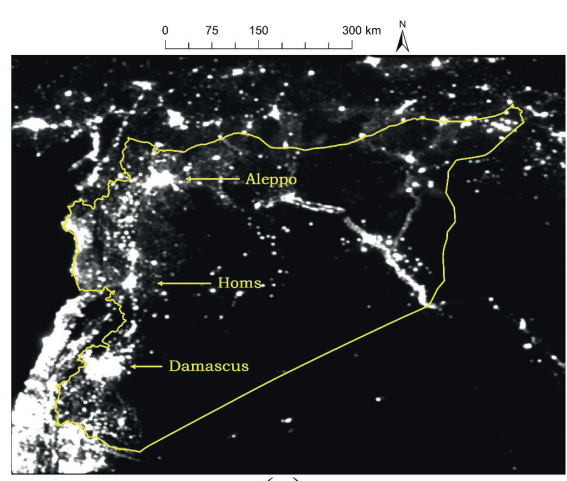

(a)

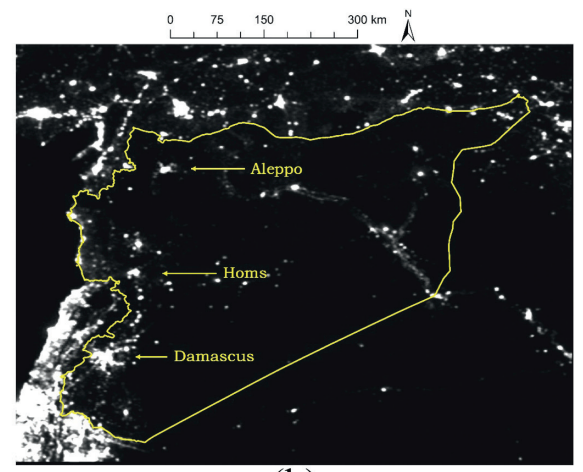

(b)

\section{FIGURE 17.8}

The DMSP/OLS NTL monthly composites in (a)March 2011 and (b)February 2014.

The results of the research on Syria were cited in a report of the United Nations Security Council. On March 11, 2015, the results were released in a telebriefing entitled "WithSyria $4^{\text {th }}$ Anniversary Campaign: Turn the Lights Back On"". On this briefing, the paper author presented the assessment images of the Syrian civil war and clarified that since the war, Syria has lost $83 \%$ of its night lights. Most areas of Syria have been plunged into darkness, with 
Aleppo Province being the most serious and the loss of night lights reaching $97 \%$. This shows that Syria is falling into a serious humanitarian disaster. This result has been reported by more than 600 media outlets and NGOs around the world, including the $7416^{\text {th }}$ meeting of United Nations Security Council, CNN, BBC, AOL, CBC, Fox News, Al Jazeera, AP, AFP, Reuters, France 24, New York Times, Guardian, Le Figaro, Jerusalem Post, etc. It can be seen from these that the night-light remote sensing can play a very important role in the assessment of humanitarian disasters.

\section{Bibliography}

[1] Sarah E. Bauer, Sara E. Wagner, Jim Burch, Rana Bayakly, and John E. Vena. A case-referent study: light at night and breast cancer risk in georgia. International Journal of Health Geographics, 12(1):23, Apr 2013.

[2] Jonathan Bennie, Thomas W Davies, James P Duffy, Richard Inger, and Kevin J Gaston. Contrasting trends in light pollution across europe based on satellite observed night time lights. Scientific reports, 4:3789, 2014.

[3] Xin Cao, Jin Chen, Hidefumi Imura, and Osamu Higashi. A svm-based method to extract urban areas from dmsp-ols and spot vgt data. Remote Sensing of Environment, 113:2205-2209, 102009.

[4] Z. Chen, B. Yu, W. Song, H. Liu, Q. Wu, K. Shi, and J. Wu. A new approach for detecting urban centers and their spatial structure with nighttime light remote sensing. IEEE Transactions on Geoscience and Remote Sensing, 55(11):6305-6319, Nov 2017.

[5] Kwangho Choi, Chung Il Lee, Kwangseok Hwang, Sang-Woo Kim, JongHwa Park, and Yeong Gong. Distribution and migration of japanese common squid, todarodes pacificus, in the southwestern part of the east (japan) sea. Fisheries Research - FISH RES, 91:281-290, 062008.

[6] Alejandro Sánchez de Miguel, Christopher CM Kyba, Martin Aubé, Jaime Zamorano, Nicolas Cardiel, Carlos Tapia, Jon Bennie, and Kevin J Gaston. Colour remote sensing of the impact of artificial light at night (i): The potential of the international space station and other dslr-based platforms. Remote Sensing of Environment, 224:92-103, 2019.

[7] Christopher NH Doll, Jan-Peter Muller, and Jeremy G Morley. Mapping regional economic activity from night-time light satellite imagery. Ecological Economics, 57(1):75-92, 2006. 
[8] Robert JR Elliott, Eric Strobl, and Puyang Sun. The local impact of typhoons on economic activity in china: A view from outer space. Journal of Urban Economics, 88:50-66, 2015.

[9] C. D. Elvidge, K. E. Baugh, S. J. Anderson, P. C. Sutton, and T. Ghosh. The night light development index (nldi): a spatially explicit measure of human development from satellite data. Social Geography, 7(1):23-35, 2012.

[10] Christopher Elvidge, Daniel Ziskin, Kimberly Baugh, Benjamin Tuttle, Tilottama Ghosh, Dee Pack, Edward Erwin, and Mikhail Zhizhin. A fifteen year record of global natural gas flaring derived from satellite data. Energies, 2(3):595-622, 2009.

[11] Christopher D Elvidge, Kimberley E Baugh, Eric A Kihn, Herbert W Kroehl, Ethan R Davis, and Chris W Davis. Relation between satellite observed visible-near infrared emissions, population, economic activity and electric power consumption. International Journal of Remote Sensing, 18(6):1373-1379, 1997.

[12] Christopher D Elvidge, Feng-Chi Hsu, Kimberly E Baugh, and Tilottama Ghosh. National trends in satellite-observed lighting. Global urban monitoring and assessment through earth observation, 23:97-118, 2014.

[13] Masanao Hara, Shuhei Okada, HIROSI YAGI, Takashi Moriyama, Koji Shigehara, and Yasuhiro Sugimori. Progress for stable artificial lights distribution extrvction accuracy and estimation of electric] power consumption by means of dmsp/ols nighttime imagery. International Journal of Remote Sensing and Earth Sciences (IJReSES), 1(1), 2010.

[14] Marc L. Imhoff, William T. Lawrence, Christopher D. Elvidge, Tera Paul, Elissa Levine, Maria V. Privalsky, and Virginia Brown. Using nighttime dmsp/ols images of city lights to estimate the impact of urban land use on soil resources in the united states. Remote Sensing of Environment, 59(1):105 - 117, 1997.

[15] Yali Katz and Noam Levin. Quantifying urban light pollution - a comparison between field measurements and eros-b imagery. Remote Sensing of Environment, 177:65 - 77, 2016.

[16] Xi Li, Xiaoling Chen, Yousong Zhao, Jia Xu, Fengrui Chen, and Hui Li. Automatic intercalibration of night-time light imagery using robust regression. Remote sensing letters, 4(1):45-54, 2013.

[17] Xi Li and Deren Li. Can night-time light images play a role in evaluating the syrian crisis? International Journal of Remote Sensing, 35(18):6648$6661,2014$. 
[18] Xi Li, Huimin Xu, Xiaoling Chen, and Chang Li. Potential of npp-viirs nighttime light imagery for modeling the regional economy of china. Remote Sensing, 5(6):3057-3081, 2013.

[19] Dengsheng Lu, Hanqin Tian, Guomo Zhou, and Hongli Ge. Regional mapping of human settlements in southeastern china with multisensor remotely sensed data. Remote Sensing of Environment, 112(9):3668$3679,2008$.

[20] Bhartendu Pandey, PK Joshi, and Karen C Seto. Monitoring urbanization dynamics in india using dmsp/ols night time lights and spot-vgt data. International Journal of Applied Earth Observation and Geoinformation, 23:49-61, 2013.

[21] Proceedings of the Asia-Pacific Advanced Network. Why viirs data are superior to dmsp for mapping nighttime lights, volume 35, 2013.

[22] Miguel O Román and Eleanor C Stokes. Holidays in lights: Tracking cultural patterns in demand for energy services. Earth's future, 3(6):182$205,2015$.

[23] Ye Wei, Hongxing Liu, Wei Song, Bailang Yu, and Chunliang Xiu. Normalization of time series dmsp-ols nighttime light images for urban growth analysis with pseudo invariant features. Landscape and Urban Planning, 128:1-13, 2014.

[24] Jiansheng Wu, Shengbin He, Jian Peng, Weifeng Li, and Xiaohong Zhong. Intercalibration of dmsp-ols night-time light data by the invariant region method. International journal of remote sensing, 34(20):7356-7368, 2013.

[25] Guo Zhang, Litao Li, Jiang Yong-hua, Xin Shen, and D Li. On-orbit relative radiometric calibration of the night-time sensor of the luojia1-01 satellite. Sensors, 18, 122018.

[26] Qingling Zhang, Bhartendu Pandey, and Karen C. Seto. A robust method to generate a consistent time series from dmsp/ols nighttime light data. IEEE Transactions on Geoscience and Remote Sensing, 54, 052016.

[27] Qingling Zhang, Crystal Schaaf, and Karen C Seto. The vegetation adjusted ntl urban index: A new approach to reduce saturation and increase variation in nighttime luminosity. Remote Sensing of Environment, 129:32-41, 2013.

[28] Qiming Zheng, Qihao Weng, Lingyan Huang, Ke Wang, Jinsong Deng, Ruowei Jiang, Ziran Ye, and Muye Gan. A new source of multi-spectral high spatial resolution night-time light imagery-j11-3b. Remote Sensing of Environment, 215, 092018. 
[29] Li Zhuo, Jin Chen, Peijun Shi, Zhihui Gu, Yida Fan, and Toshiaki Ichinose. Modeling population density of china in 1998 based on dmsp/ols nighttime light image. Acta Geographica Sinica, 60(2):266-276, 2005. 